\title{
On the Cerebral Origin of EEG Responses to TMS: Insights From Severe Cortical Lesions
}

\author{
Olivia Gosseries a,b ${ }^{\mathrm{a}}$, Simone Sarasso ${ }^{\mathrm{c}}$, Silvia Casarotto ${ }^{\mathrm{c}}$, Mélanie Boly ${ }^{\mathrm{a}}$, Caroline Schnakers ${ }^{\mathrm{a}}$, \\ Martino Napolitani ${ }^{c}$, Marie-Aurélie Bruno ${ }^{a}$, Didier Ledoux ${ }^{a}$, Jean-Flory Tshibanda ${ }^{a}$, \\ Marcello Massimini ${ }^{c, d}$, Steven Laureys ${ }^{a}$, Mario Rosanova ${ }^{\text {c,e, } *}$ \\ ${ }^{a}$ Coma Science Group, Cyclotron Research Center and Neurology Department, University and University Hospital of Liège, Liège, Belgium \\ ${ }^{\mathrm{b}}$ Postle Laboratory and Center for Sleep and Consciousness, Department of Psychiatry and Psychology, University of Wisconsin, Madison, WI, USA \\ ${ }^{\mathrm{c}}$ Department of Biomedical and Clinical Sciences "Luigi Sacco", University of Milan, Milan, Italy \\ ${ }^{\mathrm{d}}$ IRCCS Fondazione Don Carlo Gnocchi, Milan, Italy \\ ${ }^{\mathrm{e}}$ Fondazione Europea di Ricerca Biomedica, FERB Onlus, Milan, Italy
}

\section{A R T I C L E I N F O}

\section{Article history:}

Received 27 June 2014

Received in revised form

8 October 2014

Accepted 11 October 2014

Available online 4 December 2014

\section{Keywords:}

Transcranial magnetic stimulation

Electroencephalography

Brain-injury

Biological artifacts

\begin{abstract}
A B S T R A C T
Background: Transcranial magnetic stimulation combined with electroencephalography (TMS/EEG) represents a valuable tool to probe cortical excitability and connectivity. Although several procedures have been devised to abolish TMS-related artifacts, direct evidence that it is possible to record TMSevoked potentials (TEPs) that purely reflect cortical responses to TMS are still lacking.

Objective: To demonstrate that when TMS is delivered on a human head with intact nerves, scalp and ocular muscles, TEPs are present only if a functional portion of cortex is targeted and is absent otherwise. Methods: We performed extensive navigated TMS/EEG mappings in three vegetative state patients and in eight healthy controls. Patients were selected based on the extension of their cortical lesions as revealed by structural/functional imaging: the cerebral cortex was globally damaged in Patient 1 due to cerebral anoxia, Patient 2 showed a traumatic damage affecting one cerebral hemisphere, while Patient 3 was characterized by one left sided and one right-sided focal ischemic lesion.

Results: In Patient 1, TMS performed at any targeted cortical site did not elicit statistically significant TEPs. In Patient 2, TEPs were absent when the damaged hemisphere was targeted, while were present over the healthy side. In Patient 3, significant TEPs were absent when cortical lesions were targeted and present otherwise. Significant TEPs were always present in healthy controls.

Conclusions: These findings suggest that, provided that appropriate experimental procedures are employed, TEPs are genuine cortical responses detectable only when preserved cortical tissue is stimulated. Hence, a dependable assessment of cortical excitability and connectivity in brain-injured patients requires the use of neuronavigated TMS.
\end{abstract}

(c) 2015 Elsevier Inc. All rights reserved.
This study was funded by James S. McDonnell Foundation Scholar Award 2013, EU grant FP7-ICT-2011-9, n. 600806 "Corticonics" and PRIN 2010 to MM; Public Utility Foundation “Université Européenne du Travail”; Belgian National Funds for Scientific Research (FNRS); Belgian American Educational Foundation and WallonieBruxelles International. OG and MAB are Postdoctoral Researchers and SL is Research Director at FNRS. OG received support from NIH grant MH095984 to Bradley R. Postle and from Giulio Tononi.

Disclosure: the authors declare no conflict of interest.

* Corresponding author. Department of Biomedical and Clinical Sciences "L Sacco", University of Milan, Hospital Luigi Sacco, Via GB Grassi 74, Italy. Tel.: +390250319786.

E-mail address: mario.rosanova@unimi.it (M. Rosanova).

\section{Introduction}

The combination of single-pulse transcranial magnetic stimulation with electroencephalography (TMS/EEG) allows activating a selected cortical site and measuring the strength of the local and remote cortical response to this initial perturbation. Thus, to the extent that they are not confounded by electromagnetic and biological artifacts, TMS-evoked EEG potentials (TEPs) provide a direct assessment of cortical excitability and long-range cortical connectivity [1-3].

TMS-compatible EEG amplifiers have been developed to block most of the electromagnetic artifact induced by the TMS coil 
Table 1

Clinical information.

\begin{tabular}{|c|c|c|c|}
\hline & Patient 1 & Patient 2 & Patient 3 \\
\hline \multicolumn{4}{|l|}{ Clinical features } \\
\hline Gender (age, years) & Male (43) & Male (19) & Male (81) \\
\hline Cause & Cardiac arrest & Trauma & Cerebral vascular accidents \\
\hline Time since injury & 32 days & 172 days & 19 days and 5.2 years \\
\hline Diagnosis at time of TMS/EEG & VS/UWS & VS/UWS & VS/UWS \\
\hline Outcome at 12 months & GOSE 2 (i.e., still VS/UWS) & GOSE 2 (i.e., still VS/UWS) & GOSE 1 (i.e., deceased) \\
\hline \multicolumn{4}{|l|}{ Coma Recovery Scale-Revised } \\
\hline Auditory function & None & Startle reflex & None \\
\hline Visual function & None & None & None \\
\hline Motor function & Flexion to pain & Abnormal posturing & Flexion to pain \\
\hline Oromotor/verbal function & Oral reflexes & Oral reflexes & Oral reflexes \\
\hline Communication & None & None & None \\
\hline Arousal & Without stimulation & With stimulation & With stimulation \\
\hline Total score & 5 & 4 & 4 \\
\hline \multicolumn{4}{|l|}{ Electroencephalography } \\
\hline Background activity & $\begin{array}{l}\text { Low voltage diffuse, bilateral, irregular, } \\
\text { non reactive delta-theta activity. } \\
\text { No epileptic activity (essentially } \\
\text { isoelectric activity reflecting an } \\
\text { absence of electrical activity of } \\
\text { cortical origin). }\end{array}$ & $\begin{array}{l}\text { Low voltage theta-delta activity } \\
\text { with intermittent diffuse delta, } \\
\text { predominantly on the right side. } \\
\text { No epileptic activity. }\end{array}$ & $\begin{array}{l}\text { Irregular and diffuse theta-delta } \\
\text { activity with lower voltage on the left side. } \\
\text { No epileptic activity. }\end{array}$ \\
\hline \multicolumn{4}{|l|}{ Magnetic resonance imaging } \\
\hline $\mathrm{T} 1$ and FLAIR sequences & $\begin{array}{l}\text { Hyperintense lesions in caudate, } \\
\text { lenticular and thalamic nuclei especially } \\
\text { in the left pulvinar. Bilateral lesions in } \\
\text { the insula and hippocampus. Small } \\
\text { juxtacortical lesions in frontal and } \\
\text { temporal lobes. }\end{array}$ & $\begin{array}{l}\text { Bulbo-medullar junction, pons and } \\
\text { cerebellar peduncles lesions. } \\
\text { Diffuse cortico-sub-cortical lesion } \\
\text { of the left hemisphere and corpus } \\
\text { callosum. Right ventricular shunt. }\end{array}$ & $\begin{array}{l}\text { Bilateral ischemic cerebellar lesions predominantly } \\
\text { on the right side. Focal hyperintense lesions in } \\
\text { the left thalamus, in particular in the pulvinar } \\
\text { and diffuse lesion in the corpus callosum. } \\
\text { Important acute ischemic lesion in the right } \\
\text { sylvian territory and a massive subacute } \\
\text { ischemic lesion in the left sylvian territory. }\end{array}$ \\
\hline
\end{tabular}

TMS/EEG: transcranial magnetic stimulation/electroencephalography; FLAIR: Fluid Attenuated Inversion Recovery; T1: spin-lattice relaxation time; VS/UWS: vegetative state/ unresponsive wakefulness syndrome; GOSE: Extended Glasgow Outcome Scale.

discharge [4,5]; the effectiveness of these devices has been tested by recording TEPs on human as well as on phantom heads [5]. Besides the magnetic artifact, other sources, such as scalp muscle activations, eye blinks/movements, somatosensory potentials evoked by scalp stimulation, and auditory evoked potentials may contaminate the genuine cortical response to TMS [6]. These spurious activations, due to coil discharge effects on extra-cranial excitable tissues, may occur only on real heads (with intact muscles and nerves) where they are superimposed to the cortical response to TMS (intra-cranial effects); hence, they are very difficult to identify and disentangle. So far, a direct demonstration that, by employing the appropriate procedures, it is possible to record responses that are not contaminated by the extra-cranial effects of TMS (activation of scalp muscles, of somatosensory peripheral scalp nerves and activation of auditory pathways by the coil's click) is still lacking. Working under the assumption that damaged cortical tissue produces no electrical responses to a direct stimulation [7], we exploited TMS/EEG recordings performed over damaged cortical areas in brain-injured patients, in which cranial nerves and muscles are preserved.

\section{Methods}

\section{Patients enrollment and study design}

Three severely brain-injured patients diagnosed as in a vegetative state/unresponsive wakefulness syndrome (VS/UWS) on the basis of repeated behavioral evaluations with the Coma Recovery ScaleRevised (CRS-R) [8,9] were recruited for this study. Table 1 summarizes the clinical information including CRS-R total and sub-scores for the three patients at the time of TMS/EEG measurements as well as the EEG background activity and the structural lesion.

Patient 1 (male, 43 years old, anoxic etiology, 32 days postinjury) suffered a prolonged (tens of minutes) cardio-respiratory arrest. This event resulted in severe cerebral anoxia as documented by structural magnetic resonance imaging (MRI) scan (T1-weighted turbo spin-echo sequence) displaying hyperintensities localized at the level of subcortical structures [10] such as lenticular nuclei, caudate nuclei, and thalamic nuclei (Fig. 1, panel A, upper row). Accordingly, positron emission tomography (PET) scan (Fig. 1, panel A, middle and lower rows) revealed preserved glucose metabolism only in the brainstem and in the cerebellum, while cortical and thalamic structures were markedly hypometabolic [11-13]. We tested the effects of these massive bilateral cortical lesions on cortical reactivity to a direct perturbation by targeting TMS over six midline (see TMS targeting and stimulation parameters section) cortical sites encompassing the frontal and the parietal lobes of both hemispheres (left and right superior frontal gyrus, left and right precentral gyrus and left and right superior parietal cortex).

Patient 2 (male, 19 years old, traumatic etiology, 172 days postinjury) suffered head trauma. Structural MRI showed large postcontusion lesions with diffuse leucopathy confined to the left hemisphere while the right hemisphere was intact. As revealed by PET scan, lesions resulted in a hypometabolic rate encompassing the left hemisphere of the cerebral cortex (Fig. 2, panel A, upper row), while the right hemisphere was functionally preserved (Fig. 2, panel A, lower row) as compared to healthy control subjects [11-13]. In this patient, we targeted TMS over three sites of the injured hemisphere (left medial superior frontal gyrus, left posterior parietal gyrus and left superior occipital gyrus) and over three homotopic sites of the intact hemisphere (right medial superior frontal gyrus, right posterior parietal gyrus and right superior occipital gyrus).

Patient 3 (male, 81 years old, ischemic stroke, 19 days post-acute injury and 5.2 years post-subacute injury) suffered from one acute and one subacute stroke resulting, as revealed by the structural MRI, in two lesions, one affecting the right precentral gyrus and a 
A
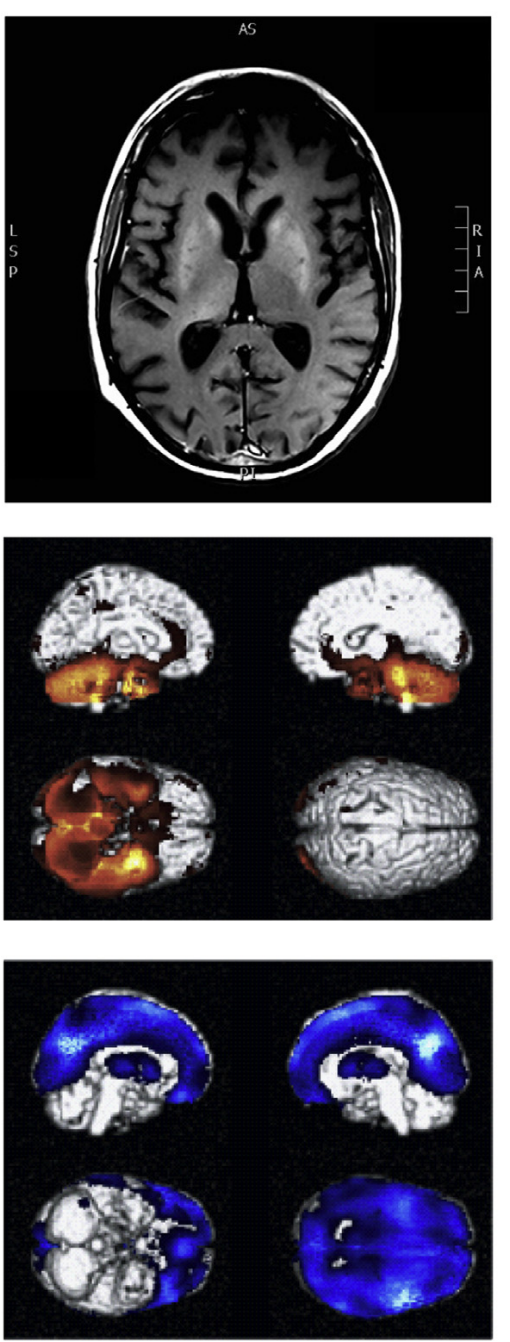

B
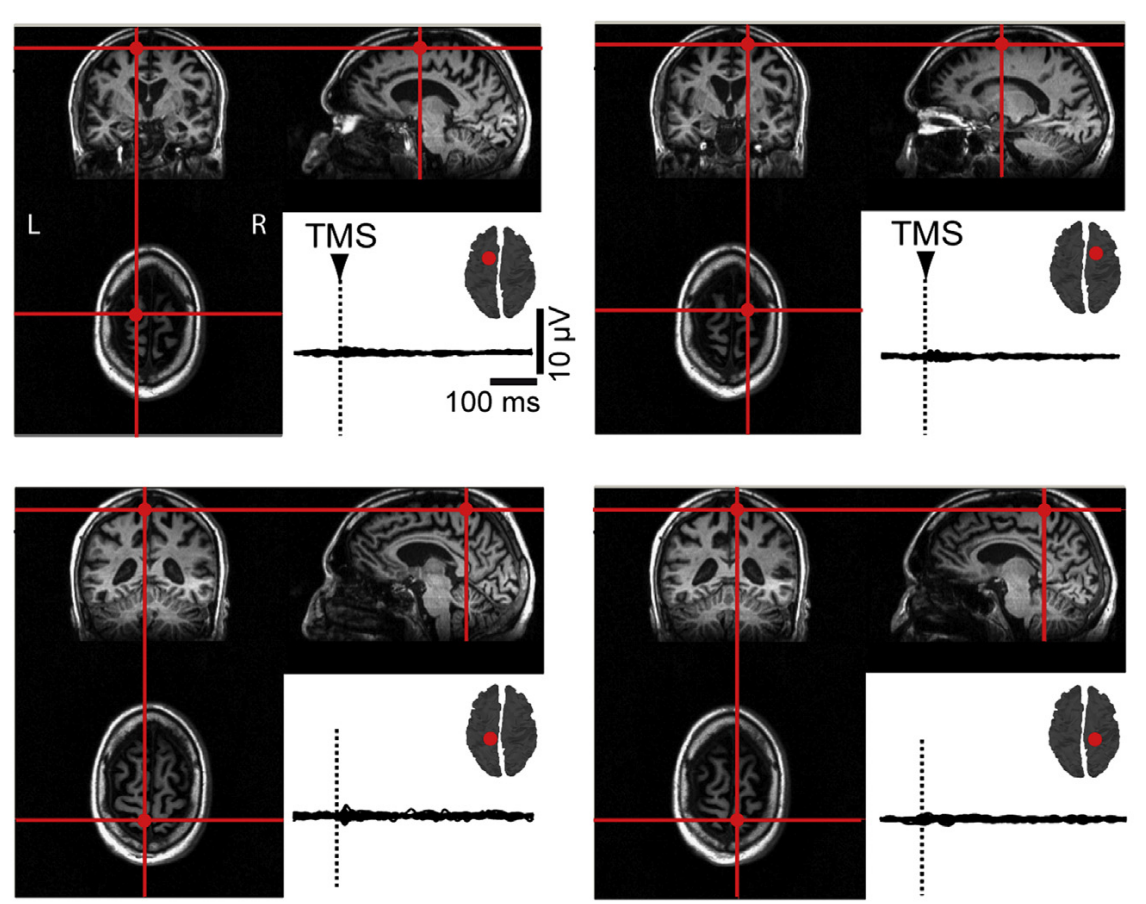

$\frac{5}{ \pm}$
$\frac{\pi}{0}$
$\frac{\pi}{\pi}$
0
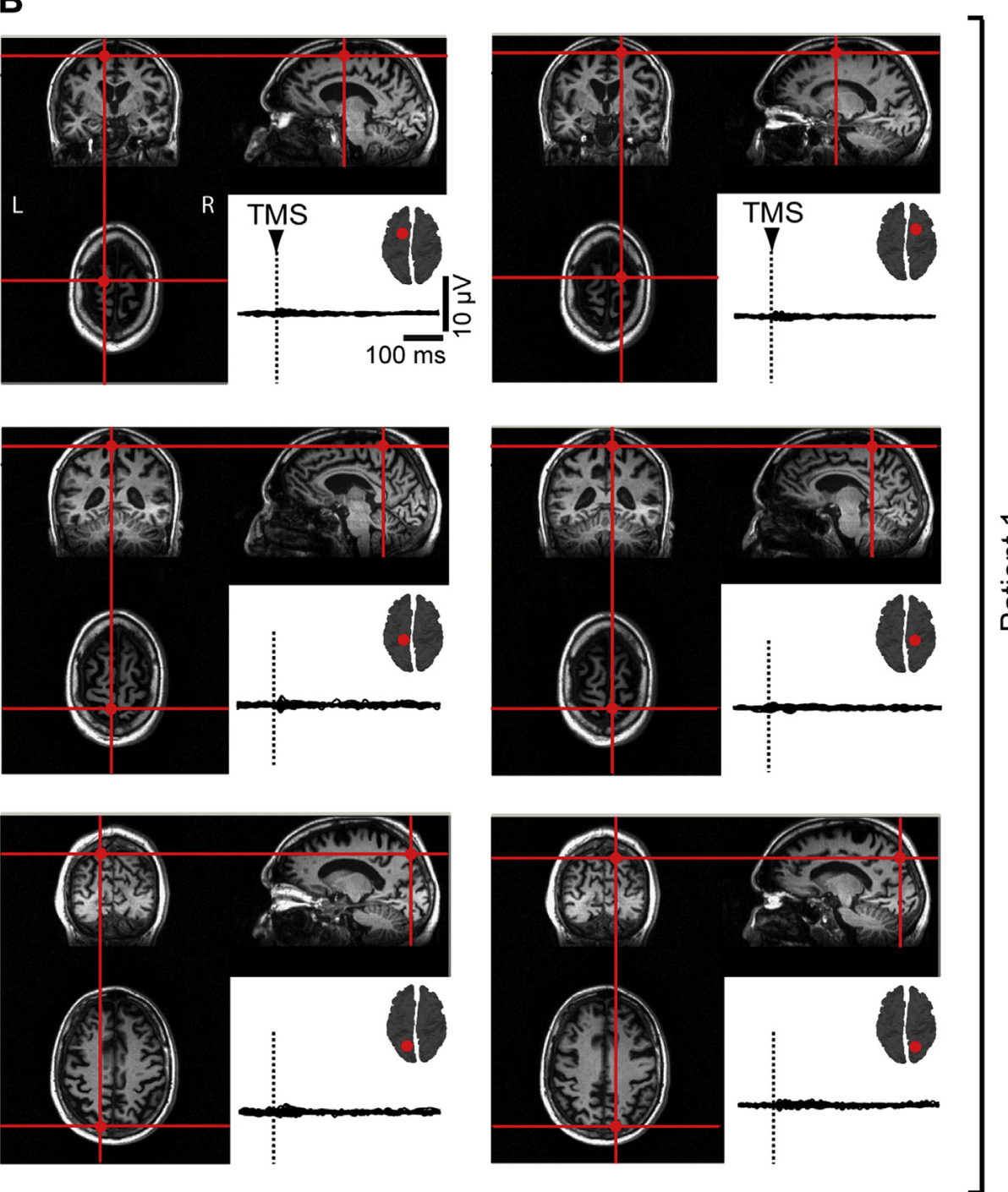

Figure 1. TEPs in diffuse anoxic cortical damage. Panel A shows a structural magnetic resonance imaging scan (MRI; T1-weighted turbo spin-echo sequence) together with positron emission tomography scans ([18 F]-fluorodeoxyglucose-PET) for Patient 1 (VS/UWS, anoxic). Panel B shows structural MRIs (T1-weighted MPRAGE sequence) and transcranial magnetic stimulation (TMS) cortical targets (red crosshairs) as estimated by the navigated brain stimulation (NBS) system in Patient 1 (VS/UWS, anoxic). TMS-evoked EEG potentials (TEPs; butterfly plots of 60 EEG channels) recorded after the stimulation of six different cortical sites are shown for each site (left and right superior frontal gyri, left and right precentral gyri and left and right superior parietal gyri). (For interpretation of the references to color in this figure legend, the reader is referred to the web version of this article.)

larger one involving most of the left sylvian territory, respectively (Fig. 3). Based on the extent and location of these lesions we targeted TMS in order to stimulate both injured and intact cortical tissue within each hemisphere. Specifically, for the right hemisphere we performed two TMS/EEG measurements within and outside the lesion involving the right precentral gyrus (Fig. 3, panel A), while for the left hemisphere we targeted TMS over four sites involving injured and intact cortical tissue of both frontal and occipital lobes (Fig. 3, panel B).

Additional TMS/EEG measurements were carried out in Patients 1 and 2 in order to assess respectively the presence of TMS-evoked muscle artifacts (by targeting a lateral right frontal site; see Supplementary Fig. 1, Panel A) and TMS-evoked auditory potentials (by performing a sham TMS with and without noise masking; see Supplementary Fig. 1, Panel B).

In addition, we performed TMS/EEG mappings in eight healthy subjects (right handed, females $n=6$, mean age $=28.6$, standard deviation $=5.09$ ) over the left, dominant hemisphere (middle or superior occipital gyrus, superior parietal gyrus, precentral gyrus and middle or caudal portion of the superior frontal gyrus). The study was approved by the Ethics Committee of the Medicine Faculty of the University of Liège and written informed consents were obtained from either the healthy subjects or by the patient's legal surrogates.

\section{TMS targeting and stimulation parameters}

All the study participants underwent one TMS/EEG session consisting of six TMS/EEG measurements for the three patients and of four TMS/EEG measurements for the healthy subjects. During each TMS/EEG measurement a different cortical site was targeted. Cortical targets were identified on MRI scans acquired on a 3T magnetic resonance scanner (Trio Tim, Siemens, Germany) using a T1-weighted MPRAGE sequence. For TMS, we employed a focal figure-of-eight coil (mean/outer winding diameter 50/70 mm, biphasic pulse shape, pulse length $280 \mu$ s, focal area of the stimulation $0.68 \mathrm{~cm}^{2}$ ) driven by a Mobile Stimulator Unit (eXimia TMS Stimulator, Nexstim Ltd.). We controlled TMS parameters by means 
A
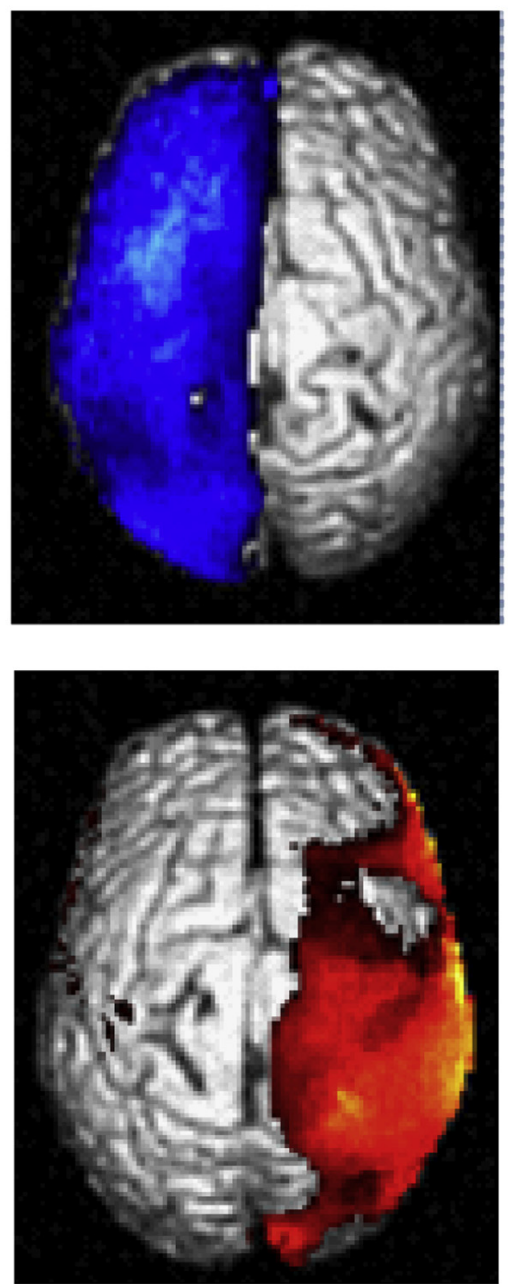

B
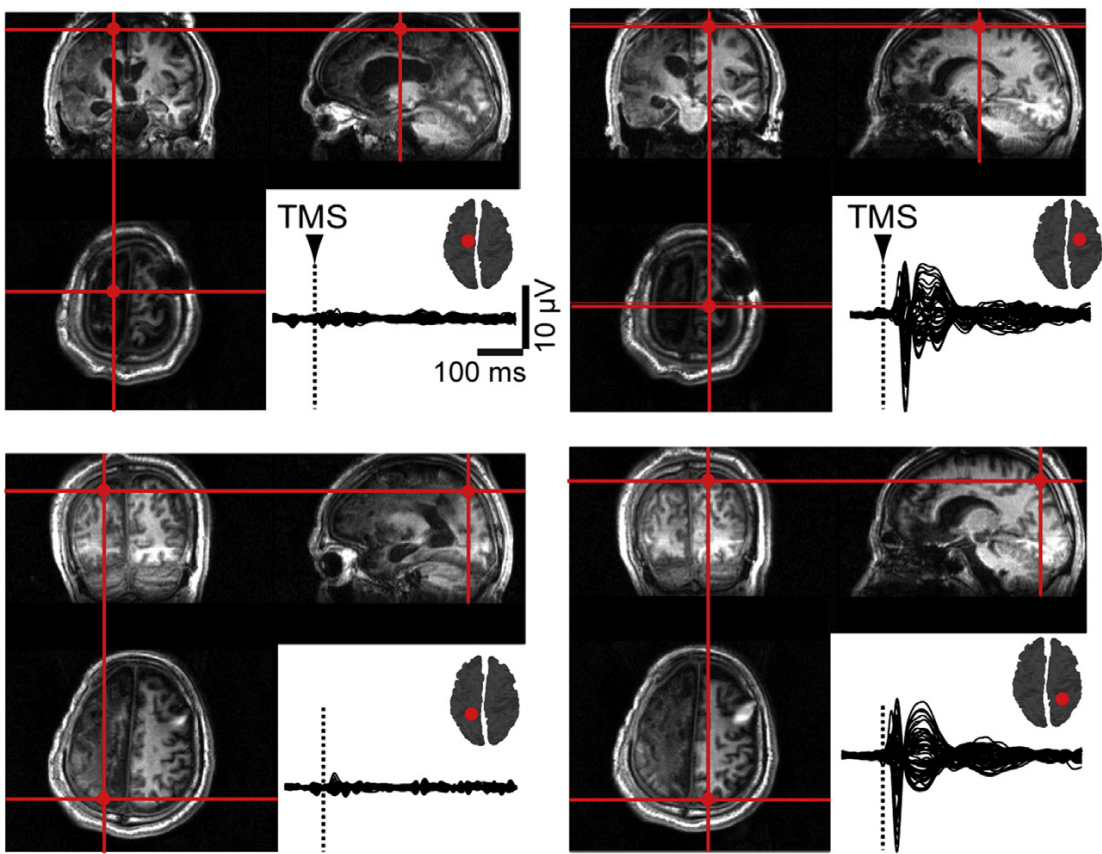

N

$+$

$\frac{\sqrt{1}}{\frac{1}{\pi}}$
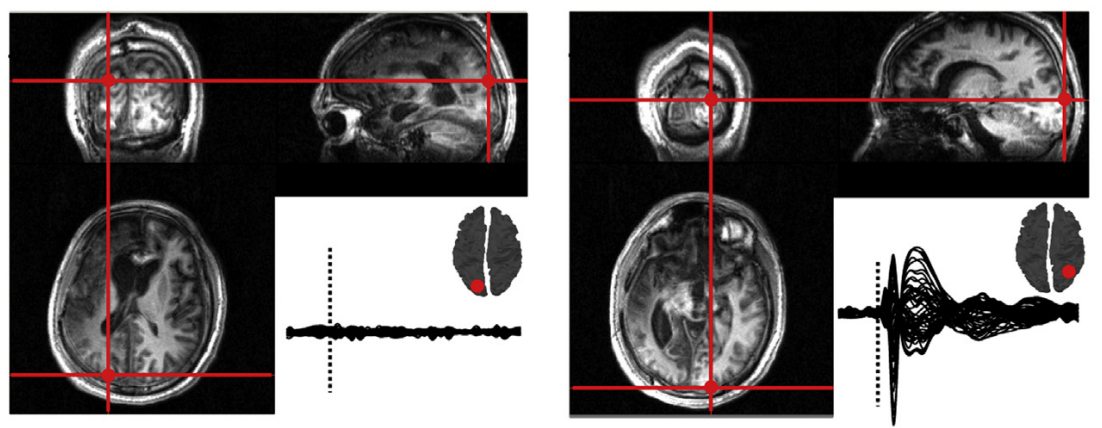

Figure 2. TEPs in diffuse unilateral traumatic cortical damage. Panel A shows PET scans of relatively preserved (orange) and hypometabolic (blue) cortical areas in Patient 2 (VS/ UWS, traumatic). Panel B shows structural MRIs (T1-weighted MPRAGE sequence) and cortical TMS targets (red crosshairs) as estimated by the NBS system together with TEPs recorded after stimulation of six cortical areas. Significant TEPs components are detected only when preserved areas are targeted and absent otherwise. (For interpretation of the references to color in this figure legend, the reader is referred to the web version of this article.)

of a navigated brain stimulation (NBS) system (Nexstim Ltd.) employing a 3D infrared tracking position sensor unit to locate the relative positions of the coil and subject's head within the reference space of individual MRI scans. NBS system also estimated, online, the distribution and the intensity (expressed in $\mathrm{V} / \mathrm{m}$ ) of the intracranial electric field induced by TMS. The location of the maximum electric field induced by TMS on the cortical surface (hot spot) was always kept on the convexity of the targeted gyrus with the induced current perpendicular to its main axis. The coil was always placed on a scalp area as close as possible to the midline, far away from facial or temporal muscles and nerve endings [14], except for two TMS/EEG measurements in which we aimed at stimulating lateral areas of the frontal lobe (see Supplementary Fig. 1, panel A for Patient 1 and Fig. 3, panel A, lower row for Patient 3). At least 300 trials were collected for each stimulation site. TMS was delivered with an interstimulus interval jittering randomly between 2000 and $2300 \mathrm{~ms}(0.4-0.5 \mathrm{~Hz})$. Before every TMS/EEG measurement the output of the stimulating unit was adjusted to induce an electric field of $140 \mathrm{~V} / \mathrm{m}$ on the cortical surface (as estimated by the NBS), an intensity that is largely above the threshold $(50 \mathrm{~V} / \mathrm{m})$ for a significant EEG response [15-17]. By employing the MRI-guided NBS system we were able to precisely target cortical sites within and outside lesions. TMS sham stimulation was performed by tilting the stimulation coil at $90^{\circ}$ over the head vertex.

\section{EEG recordings}

TMS/EEG measurements were performed using a TMS-compatible 60-channel EEG amplifier (Nexstim Ltd.). This device prevents amplifier saturation and reduces, or abolishes, the magnetic artifacts induced by the coil's discharge via a sample-and-hold circuit activated about $50 \mu$ s before the TMS pulse and released $2.5 \mathrm{~ms}$ after [5]. To further optimize TMS compatibility, the impedance at all electrodes was kept below $5 \mathrm{k} \Omega$. EEG signals were referenced to an additional electrode on the forehead, filtered $(0.1-350 \mathrm{~Hz})$ and sampled at $1450 \mathrm{~Hz}$. Two extra sensors were used to record the electrooculogram. Moreover, as in previous works [7,17-19]. study participants wore inserted earplugs through which a noise masking, reproducing the time-varying frequency components of the TMS-associated "click", was played throughout each TMS/EEG session in order to prevent "click"-induced EEG auditory evoked potentials [20] as well as eye blinks or eye muscle reactions [1,6]. Additionally, the bone conduction produced by the mechanical vibration of the TMS was minimized by placing a thin foam layer between the coil and the EEG cap [21].

\section{General experimental procedures}

During each TMS/EEG measurement, study participants were lying on their bed (patients) or sitting on a reclining chair (healthy 

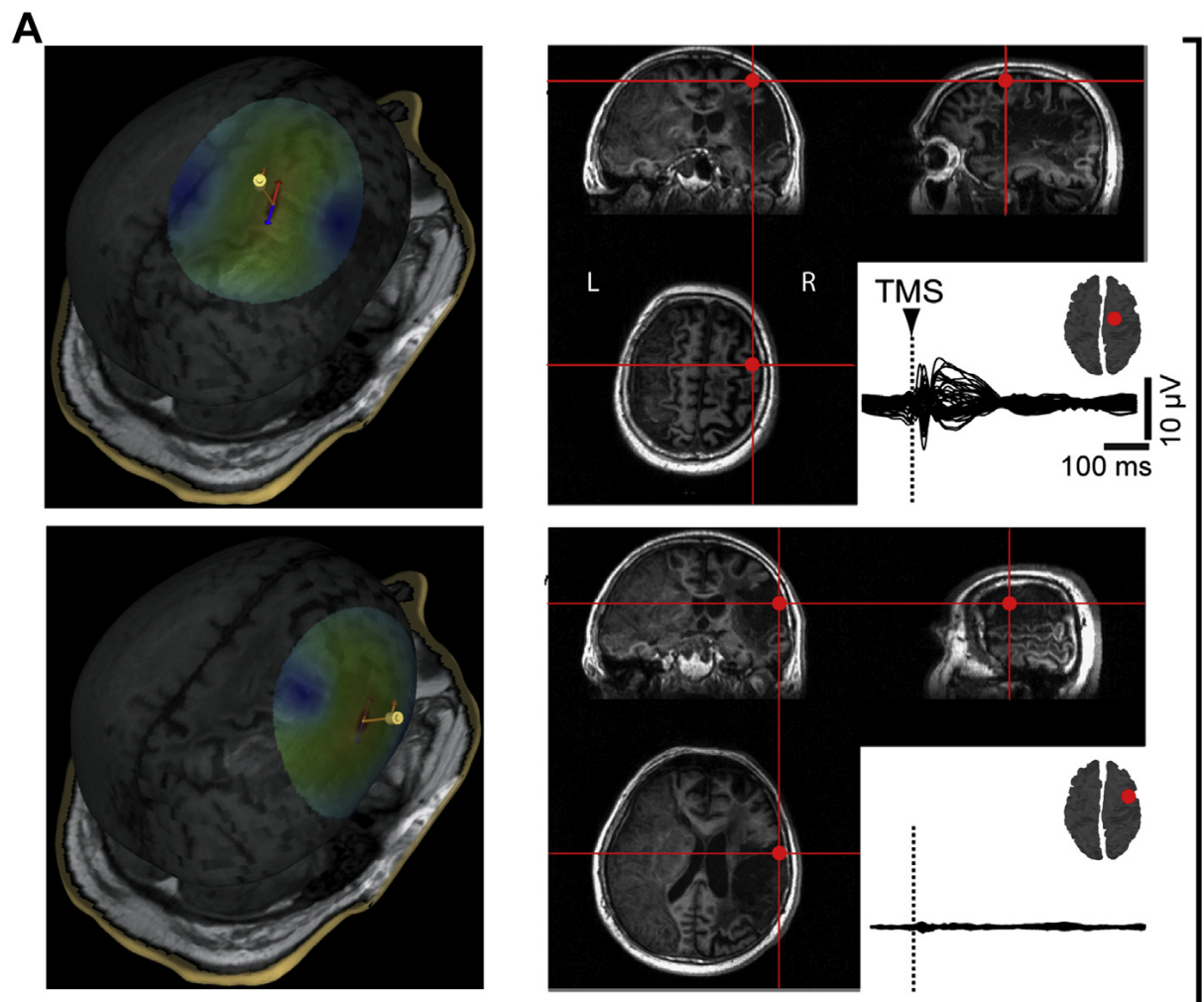

B
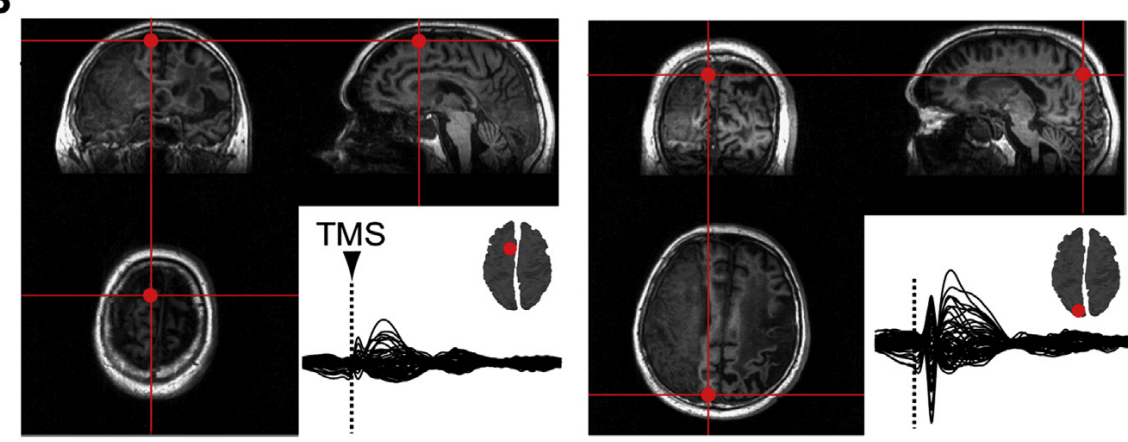

$m$
$\frac{1}{0}$
$\frac{1}{\pi}$
$\frac{1}{n}$
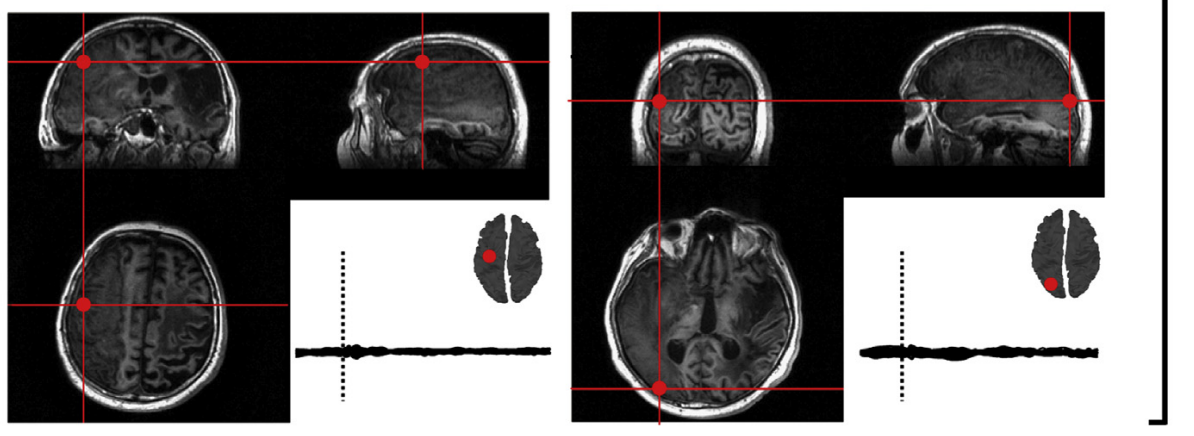

Figure 3. TEPs in cortical focal ischemia. Panel A shows two TMS cortical targets (one within and one outside a focal ischemic lesion in Patient 3 ) as displayed by the NBS software that was employed in the present study (see Methods section). The corresponding MRI scans and TEPs are also shown. Panel B shows further examples of TEPs recorded when TMS was targeted within and outside ischemic cortical lesions.

subjects), awake and with their eyes open. Patients were recorded without sedation and in case of behavioral signs of drowsiness (e.g. closure of eyelids), recordings were momentarily interrupted and they were stimulated using the CRS-R arousal facilitation protocols [8]. The stability of the stimulation coordinates was continuously monitored throughout all acquisitions by means of the NBS system. If the displacement from the cortical target was greater than $4 \mathrm{~mm}$, the TMS/EEG measurement was interrupted and the coil was repositioned. At the end of the experiment, the stimulation coordinates were recorded and the electrode positions were digitized.

\section{Data analysis and statistics}

Data analysis was performed using Matlab R2006a (The MathWorks). First, TMS/EEG trials containing noise, spontaneous eye blinks, eye movements or muscle artifacts were detected and rejected using an automatic algorithm [15]. Then, EEG data were 

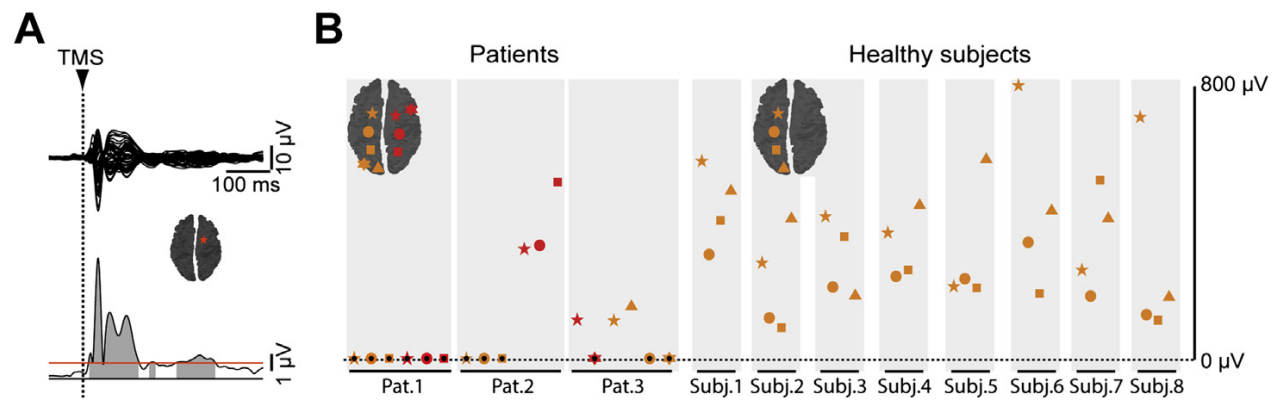

Figure 4. Global cortical reactivity values for patients and healthy subjects. In Panel A, the procedure to detect the presence of a statistically significant TMS-evoked EEG response is outlined. An example of butterfly plot (upper row) together with the corresponding Global Mean Field Power (GMFP) (lower row) and the stimulation site (red star on the gray cortical map) are reported. To assess the threshold for significance $(P<0.01)$ of GMFP time-series (red line), a bootstrap method was applied (see Methods section). For each GMFP time-series, only the significant voltage values were cumulated over the first $300 \mathrm{~ms}$ post-stimulus. The response was considered non-significant whenever this procedure failed to reveal any significant value within the post-stimulus interval (0-300 ms). This resulted in a single, global index of cortical reactivity for each TMS/EEG measurement performed in patients and healthy subjects (Panel B). Black dots within markers identify damaged sites of stimulation. (For interpretation of the references to color in this figure legend, the reader is referred to the web version of this article.)

average referenced, down-sampled to half of the original sampling rate $(725 \mathrm{~Hz})$, band pass filtered $(1-80 \mathrm{~Hz})$ and baseline corrected over $300 \mathrm{~ms}$ pre-stimulus. Each TMS-evoked response was obtained by averaging $150-250$ artifact-free trials.

In order to quantify cortical reactivity, we measured the overall amount of electrical activity induced by TMS by calculating the Global Mean Field Power (GMFP) [22] from the multichannel averaged signals as follows:

$\operatorname{GMFP}(t)=\sqrt{\frac{\sum_{i}^{k}\left(V_{i}(t)-V_{\text {mean }}(t)\right)^{2}}{k}}$

where $k$ is the number of channels, $V_{i}$ is the voltage measured with channel $i$, and $V_{\text {mean }}$ is the mean of the measured voltages across channels.

To assess the threshold for significance of GMFP time-series, a bootstrap method [23-25], which does not assume normal distribution of the observations, was applied by shuffling the time samples of pre-stimulus activity (from -300 to $-50 \mathrm{~ms}$ ) at the single-trial level and by calculating 500 surrogated pre-stimulus GMFP time-series. From each random realization, the maximum value across all latencies was selected to obtain a maximum distribution (control for type I error) and significance level was set at $P<0.01$. For each GMFP time-series, the significant voltage values were cumulated over the first $300 \mathrm{~ms}$ post-stimulus. This resulted in a single, global index of cortical reactivity for each TMS/EEG measurement in both patients and healthy subjects [7,19]. On the contrary, the response was considered non-significant whenever this procedure failed to reveal any significant value within the poststimulus interval $(0-300 \mathrm{~ms})$.

\section{PET measurements}

In Patients 1 and 2 , cerebral metabolic rates for glucose were measured by means of PET scan. Patients were monitored by two anesthesiologists throughout the procedure. [18 F]fluorodeoxyglucose-PET (FDG-PET) data were acquired after intravenous injection of $300 \mathrm{MBq}$ on a Philips Gemini TF PET-CT scanner at the University Hospital of Liege, Belgium. Data were spatially normalized, smoothed $(14 \mathrm{~mm}$ full width at a half maximum) and analyzed using Statistical Parametric Mapping (SPM8; www.fil.ion.ucl.ac.uk/spm). The analysis identified brain regions with significant decreased metabolism in patients as compared to controls. The design matrix included the patients' scan and the 39 control subjects' scans with global normalization performed by proportional scaling [2]. All results were corrected for multiple comparisons and considered significant at false discovery rate $P<0.05$ (corrected).

\section{Results}

As shown in Fig. 1 (panel B), stimulations performed in Patient 1 (presenting cortical death caused by anoxia, as documented by MRI and PET scans, Fig. 1, panel A) failed to elicit a significant EEG response at the electrode under the coil as well as at distant electrodes (Fig. 4, panel B). This was the case for all six targeted cortical sites, suggesting that anoxia resulted in a widespread obliteration of cortical responsiveness. Notably, TMS applied over a lateral portion of the right frontal lobe resulted in a very large $(>300 \mu \mathrm{V})$ biphasic EEG response confined to the stimulated area (Supplementary Fig. 1, panel A) and consistent with a TMS-evoked scalp muscle activation [14].

While the absence of response observed in Patient 1 tests for the presence of extra-cranial sources of artifact (direct scalp and ocular muscle activation), it does not necessarily rules out the presence of cortical sources that may be accidentally activated by the peripheral effects of TMS (somatosensory evoked potentials elicited through scalp nerves and auditory evoked potentials elicited by the coil's "click"). Hence, the same experiment was repeated in Patient 2, who suffered from a head trauma, in whom structural imaging (MRI, Fig. 2, panel B) as well as functional imaging (PET, Fig. 2, panel A) showed a diffuse damage of the left hemisphere and no structural/metabolic alteration in the right hemisphere. Crucially, in this patient, sensory afferents from the left hemi-soma to the right hemisphere were also preserved as suggested by the presence of somatosensory evoked potentials (SEPs) following left median nerve stimulation (N20 at $23 \mathrm{~ms}$; data not shown). Also, sham TMS stimulation without noise masking (Supplementary Fig. 1, right panel B) resulted in a significant evoked potential demonstrating the integrity of auditory pathways and cortices. Similar to the stimulations performed in Patient 1, Fig. 2 (panel B, left column) shows that when three left-sided cortical sites (medial superior frontal gyrus, posterior parietal gyrus and superior occipital gyrus) were stimulated, TMS did not trigger any significant response neither at the electrode under the coil nor at distant sites (Fig. 4, panel B). Conversely, when the three homologous, preserved, rightsided cortical areas were targeted, TMS triggered significant EEG responses across all stimulation sites (Fig. 2, panel B, right column; Fig. 4, panel B). In this case, TMS-evoked slow, positive-negative EEG responses closely resembling those observed during physiological - deep sleep [19] and pharmacological anesthesia [18] - loss of consciousness. These responses were also recently reported to be 
systematically associated with VS/UWS condition due to traumatic or vascular events [7]. Thus, the absence of significant EEG responses in the electrodes over the healthy (right) hemisphere when TMS was applied on the damaged (left) hemisphere rules out the contribution of somatosensory/auditory activations to TMS-evoked EEG responses.

We additionally explored the genuine cortical origin of TEPs in Patient 3 who suffered from one acute and one subacute cortical stroke that resulted, as revealed by the structural MRI, in two lesions: one affecting the right precentral gyrus and a larger one involving most of the left sylvian territory (Fig. 3). As for Patient 2, slow, positive-negative significant EEG responses to TMS were evoked only when preserved cortical sites of both hemispheres were targeted (Fig. 3, panels A and B, upper rows; Fig. 4, panel B) and were absent otherwise (Fig. 3, panels A and B, lower rows; Fig. 4, panel B). Notably, in Patient 3 this contrast was evident within the same hemisphere, when TMS was targeted over two relatively close cortical sites within and outside a lesion (Fig. 3, panel A).

We finally compared the global cortical reactivity derived from TMS/EEG measurements across patients and healthy subjects (overall $50 \mathrm{TMS} / \mathrm{EEG}$ measurements in 11 study participants) by plotting the cumulated significant GMFP time points (see Methods section and Fig. 4, panel A). In line with the abovementioned results, cortical reactivity was abolished (cumulated GMFP $=0$ ) whenever TMS was targeted over a damaged cortical area (Fig. 4, panel $\mathrm{B}$, patients plot). On the contrary, cortical reactivity was preserved in patients (cumulated GMFP $>0$ ) only when TMS was targeted over healthy cortical sites and in healthy subjects (Fig. 4, panel B). The range of these positive cumulated GMFP values in patients was overlapping with the one obtained in healthy subjects.

\section{Discussion}

In this study, we tested the hypothesis that EEG responses to TMS are absent when stimulating a damaged portion of the cortical mantle and present otherwise, provided that proper experimental procedures are applied, such as playing noise masking and stimulating midline scalp sites. The absence of EEG responses to TMS when stimulating injured tissue would corroborate the notion that TEPs, when present, purely reflect the direct activation of the targeted cortical sources, hence are unconfounded by the peripheral activation of scalp muscles and auditory/somatosensory cortices. To this aim, we performed extensive (at least six cortical sites per patient) TMS/EEG mappings during wakefulness in three braininjured patients with a diagnosis of VS/UWS [26,27]. The three patients were recruited based on the extent of the cortical lesions: cerebral cortex was globally damaged in Patient 1 due to prolonged anoxia, one cerebral hemisphere was damaged in Patient 2 due to a traumatic event while Patient 3 was characterized by multiple cortical lesions due to vascular events involving both hemispheres. By means of a navigation system patients were stimulated systematically within and outside cortical lesions and the presence of statistically significant EEG responses was detected. We compared these results with the ones obtained after performing extensive TMS/EEG mappings in eight healthy subjects.

Results show that TMS only leads to significant EEG responses when functionally and structurally preserved brain areas are targeted. We conclude that, when the appropriate recording procedure is employed, TEPs are not confounded by eye, muscles, somatosensory or auditory artifacts and, hence, reflect genuine cortical responses to a direct cortical stimulation.

In principle, TMS/EEG measurements can be corrupted by both electromagnetic and biological artifacts. Indeed, recording EEG responses under the influence of strong electromagnetic fields induced by TMS represents a technical as well as a methodological challenge [6]. It has been shown that TEPs can be affected by early large amplitude electromagnetic artifacts lasting for tens of milliseconds [28]. This technical problem has been successfully solved by the development of fully TMS-compatible EEG amplifiers [5]. Besides the magnetic artifact, spurious biological activations may still contaminate the genuine cortical response to TMS. For instance, the sound produced by the TMS discharge ("click") can evoke an auditory response which can be effectively abolished by employing a masking sound reproducing the time-varying frequency components of the TMS "click" [19].

Other biological events such as muscle activations or eye blinks and movements can be triggered by the TMS discharge and, hence, produce a misleading interpretation of TEPs. TMS can also evoke large EEG responses by directly stimulating scalp muscles [14]. In the present study, we ask to what extent these confounding factors could contaminate TEPs by performing extensive TMS/EEG mappings in awake, VS/UWS patients affected by severe cortical lesions. As an example, the absence of any significant TMS-evoked EEG deflection following midline TMS performed in post-anoxic Patient 1 , together with the presence of excitable scalp muscles (as shown by direct muscle stimulation in Supplementary Fig. 1, panel A) corroborates the idea that TMS/EEG recordings are normally unconfounded by scalp and eye muscles activations.

In principle, TMS can also produce a direct activation of somatosensory scalp nerve endings, possibly resulting in the generation of SEPs. In Patient 2, a traumatic event massively damaged the left hemisphere leaving the right hemisphere intact. Importantly, when the left median nerve was stimulated electrically, normal SEPs were recorded over the right side of the scalp (suggesting that somatosensory pathways and cortices were preserved). If TEPs were contaminated by SEPs, TMS of the left side of the scalp would have elicited a significant, right-sided EEG response. The absence of significant SEPs suggests that the TMS activation of trigeminal fibers innervating the scalp is not effective in eliciting a cortical response that is visible in the EEG. In addition, the failure of TMS in triggering any significant EEG response when applied over the damaged hemisphere further suggests that the masking sound successfully abolished TMS-evoked auditory potentials [15,17] otherwise present in this patient (see Supplementary Fig. 1, Panel B). This procedure is also effective in preventing TMS-evoked startle responses [29].

TMS/EEG measurements performed in Patient 3, whose cerebral cortex had two focal ischemic lesions, confirmed that the effects of TMS are eminently mediated by its impact on cortical excitable tissues rather than by its peripheral effects. In this patient, employing a neuronavigation system, we were able to systematically target TMS within and, a few centimeters apart, outside cortical lesions. In the former case, TMS did not trigger any significant EEG response, while significant EEG responses were recorded when TMS was targeted outside a cortical lesion. This finding also mandates the use of an MRI/CT-guided neuronavigation system in brain-damaged patients in order to achieve an accurate targeting of the intact cortical areas, thus avoiding massive as well as focal cortical lesions and reducing the rate of false negative results in the assessment of cortical excitability in brain-injured patients.

Overall, the present experiments provide empirical evidence that, when confounding factors are appropriately controlled for, TEPs purely reflect the response of the cortical tissue to TMS. The proper experimental procedures should include: 1) employing neuronavigation to maximize the direct effect of TMS on the underlined cortical tissue, thus reducing the need for high stimulation intensities; 2) targeting central sites of the scalp in order to minimize the likelihood of muscle and/or nerve endings direct 
activation [14]; 3) playing noise masking through inserted earplugs in order to prevent auditory potentials evoked by the TMS "click."

\section{Acknowledgment}

The authors thank Athena Demertzi (Coma Science Group) for her help.

\section{Supplementary data}

Supplementary data related to this article can be found at http:// dx.doi.org/10.1016/j.brs.2014.10.008.

\section{References}

[1] Miniussi C, Thut G. Combining TMS and EEG offers new prospects in cognitive neuroscience. Brain Topogr 2010;22:249-56.

[2] Rogasch NC, Fitzgerald PB. Assessing cortical network properties using TMSEEG. Hum Brain Mapp 2013;34:1652-69.

[3] Ziemann U. Transcranial magnetic stimulation at the interface with other techniques: a powerful tool for studying the human cortex. Neuroscientist 2011:17:368-81.

[4] Ilmoniemi RJ, Virtanen J, Ruohonen J, et al. Neuronal responses to magnetic stimulation reveal cortical reactivity and connectivity. Neuroreport 1997;8:3537-40.

[5] Virtanen J, Ruohonen J, Näätänen R, Ilmoniemi RJ. Instrumentation for the measurement of electric brain responses to transcranial magnetic stimulation. Med Biol Eng Comput 1999;37:322-6.

[6] Ilmoniemi RJ, Kičić D. Methodology for combined TMS and EEG. Brain Topogr 2010;22:233-48.

[7] Rosanova M, Gosseries O, Casarotto S, et al. Recovery of cortical effective connectivity and recovery of consciousness in vegetative patients. Brain 2012; 135:1308-20.

[8] Giacino JT, Kalmar K, Whyte J. The JFK Coma Recovery Scale-Revised: measurement characteristics and diagnostic utility. Arch Phys Med Rehabil 2004;85:2020-9.

[9] Schnakers C, Majerus S, Giacino J, et al. A French validation study of the Coma Recovery Scale-Revised (CRS-R). Brain Inj 2008;22:786-92.

[10] Ammermann H, Kassubek J, Lotze M, et al. MRI brain lesion patterns in patients in anoxia-induced vegetative state. J Neurol Sci 2007;260:65-70.
[11] Bruno MA, Majerus S, Boly M, et al. Functional neuroanatomy underlying the clinical subcategorization of minimally conscious state patients. J Neurol 2012;259:1087-98

[12] Bruno MA, Vanhaudenhuyse A, Schnakers C, et al. Visual fixation in the vegetative state: an observational case series PET study. BMC Neurol 2010;10:35.

[13] Thibaut A, Bruno MA, Chatelle C, et al. Metabolic activity in external and internal awareness networks in severely brain-damaged patients. J Rehabil Med 2012;44:487-94.

[14] Mutanen T, Maki H, Ilmoniemi RJ. The effect of stimulus parameters on TMSEEG muscle artifacts. Brain Stimul 2012;6:371-6.

[15] Casali AG, Casarotto S, Rosanova M, Mariotti M, Massimini M. General indices to characterize the electrical response of the cerebral cortex to TMS. Neuroimage 2010;49:1459-68.

[16] Komssi S, Savolainen P, Heiskala J, Kahkonen S. Excitation threshold of the motor cortex estimated with transcranial magnetic stimulation electroencephalography. Neuroreport 2007;18:13-6.

[17] Rosanova M, Casali A, Bellina V, Resta F, Mariotti M, Massimini M. Natural frequencies of human corticothalamic circuits. J Neurosci 2009;29:7679-85.

[18] Ferrarelli F, Massimini M, Sarasso S, et al. Breakdown in cortical effective connectivity during midazolam-induced loss of consciousness. Proc Natl Acad U S A 2010;107:2681-6.

[19] Massimini M, Ferrarelli F, Huber R, Esser SK, Singh H, Tononi G. Breakdown of cortical effective connectivity during sleep. Science 2005;309:2228-32.

[20] Nikouline V, Ruohonen J, Ilmoniemi R. The role of the coil click in TMS assessed with simultaneous EEG. Clin Neurophysiol 1999;110:1325-8.

[21] Ter Braack EM, de Vos CC, van Putten JAM. Masking the auditory evoked potential in TMS-EEG: a comparison of various methods. Brain Topogr 2013. http://dx.doi.org/10.1007/s10548-013-0312-z. [Epub ahead of print].

[22] Lehmann D, Skrandies W. Reference-free identification of components of checkerboard-evoked multichannel potential fields. Electroencephalogr Clin Neurophysiol 1980;48:609-21.

[23] Delorme A, Makeig S. EEGLAB: an open source toolbox for analysis of singletrial EEG dynamics including independent component analysis. J Neurosci Methods 2004;134:9-21.

[24] Lv J, Simpson DM, Bell SL. Objective detection of evoked potentials using a bootstrap technique. Med Eng Phys 2007;29:191-8.

[25] McCubbin J, Yee T, Vrba J, et al. Bootstrap significance of low SNR evoked response. J Neurosci Methods 2008;168:265-72.

[26] Laureys S, Celesia GG, Cohadon F, et al. Unresponsive wakefulness syndrome: a new name for the vegetative state or apallic syndrome. BMC Med 2010;8:68.

[27] Laureys S, Owen AM, Schiff ND. Brain function in coma, vegetative state, and related disorders. Lancet Neurol 2004;3:537-46.

[28] Veniero D, Bortoletto M, Miniussi C. TMS-EEG co-registration: on TMSinduced artifact. Clin Neurophysiol 2009;120:1392-9.

[29] Corthout E, Uttl B, Juan CH, Hallett M, Cowey A. Suppression of vision by transcranial magnetic stimulation: a third mechanism. Neuroreport 2000;11:2345-9. 